Research Paper

\title{
HN125: A Novel Immunoadhesin Targeting MUC16 with Potential for Cancer Therapy
}

\author{
Xinran Xiang1', Mingqian Feng ${ }^{1}$, Mildred Felder2 ${ }^{2}$, Joseph P. Connor ${ }^{2}$, Yan-gao Man ${ }^{3}$, Manish S. Patankar ${ }^{2}$ \\ and Mitchell $\mathrm{Ho}^{1 凶}$
}

1. Antibody Therapy Unit, Laboratory of Molecular Biology, Center for Cancer Research, National Cancer Institute, National Institutes of Health, Bethesda, Maryland 20892, USA;

2. Department of Obstetrics and Gynecology, University of Wisconsin-Madison, Madison, Wisconsin 53792, USA;

3. Department of Gynecologic and Breast Pathology, Armed Forces Institute of Pathology and American Registry of Pathology, Washington, DC 20306, USA.

Corresponding author: Mitchell Ho, PhD, Antibody Therapy Unit, Laboratory of Molecular Biology, Center for Cancer Research, National Cancer Institute, 37 Convent Drive, Room 5002C, Bethesda, MD 20892-4264. Web site: http:/ / ccr.cancer.gov/staff/staff.asp?profileid=7668; Phone: (301) 451-8727; Fax: (301) 402-1344; E-mail: homi@mail.nih.gov

(C) Ivyspring International Publisher. This is an open-access article distributed under the terms of the Creative Commons License (http://creativecommons.org/ licenses/by-nc-nd/3.0/). Reproduction is permitted for personal, noncommercial use, provided that the article is in whole, unmodified, and properly cited.

Received: 2011.04.20; Accepted: 2011.05.16; Published: 2011.05.16

\begin{abstract}
Background: The mucin MUCl6 expresses the repeating peptide epitope CAI25 that has been known for decades to be a well-validated cancer marker that is overexpressed on the cell surface of ovarian cancers and other malignant tumors. In spite of recent efforts to make mouse monoclonal antibodies to MUCI6 to treat ovarian cancer, a human monoclonal antibody against this mucin has not been described. MUCI 6 interacts with mesothelin, a protein that mediates heterotypic cancer cell adhesion, indicating that $\mathrm{MUCl} 6$ and mesothelin play an important role in the peritoneal implantation and metastasis of ovarian tumors. Therefore, a suitable candidate for therapeutic targeting of $\mathrm{MUCl} 6$ would functionally block the interaction of MUCI6 and mesothelin.

Methodology/Principal Findings: Here we report the generation of a novel immunoadhesin, $\mathrm{HNI} 25$, against $\mathrm{MUCl} 6$ that consists of a functional MUCl 6 binding domain of mesothelin $(I A B)$ and the Fc portion of a human antibody $\operatorname{lgGI}$. The yield for purified HNI25 proteins is over $100 \mu \mathrm{g} / \mathrm{mL}$ of HEK-293 culture supernatant. We show that HNI 25 has high and specific affinity for MUCl6-expressing cancer cells by flow cytometry and immunohistochemistry. $\mathrm{HNI} 25$ has the ability to disrupt the heterotypic cancer cell adhesion mediated by the MUCI6-mesothelin interaction. Moreover, it elicits strong antibody-dependent cell mediated cytotoxicity against MUCI6-positive cancer cells in vitro.

Conclusion/Significance: This report describes a novel human immunotherapeutic agent highly specific for MUCl6 with potential for treating ovarian cancer and other MUCI6-expressing tumors. Because of its lower immunogenicity in patients, a fully human protein is the most desirable format for clinical applications. We believe that the methods developed here may apply to the generation of other tumor-targeting immunoadhesins when it is difficult to obtain a human monoclonal antibody to a given antigen for clinical applications. The resultant immunoadhesins can have advantages usually found in monoclonal antibodies such as ease of purification, high binding affinity and effector functions.
\end{abstract}

Key words: immunoadhesin, human Fc fusion, mesothelin, mucin MUC16/CA125, antibody-dependent cellular cytotoxicity (ADCC), ovarian cancer, mesothelioma. 


\section{INTRODUCTION}

Epithelial ovarian cancer is the most lethal gynecologic malignancy in the United States, resulting in an estimated 21,880 new cases and 13,850 deaths in $2010[1,2]$. The high mortality rate is due to the lack of effective screening methods and the high incidence of chemotherapy resistance. Targeted immunotherapy with monoclonal antibodies (mAbs) has become a promising strategy for cancer treatment. A mAb can selectively target tumor cells that express tumor-specific antigens but not normal healthy cells. Novel mAbs have become one of the biggest classes of new drugs approved for the treatment of cancer, including ovarian cancer. Hundreds of mAbs, as well as novel $F_{c}$ fusion proteins that are composed of binding peptides or proteins fused to the Fc domain of immunoglobulin G, are undergoing clinical study [3]. By the end of 2010, a total of 30 of these candidates $(25$ $\mathrm{mAbs}$ and $5 \mathrm{Fc}$ fusion proteins) were in Phase $2 / 3$ or Phase 3 clinical studies [3]. Many therapeutic mAbs against vascular endothelial growth factor (VEGF) or VEGF receptor (VEGFR) [4-6], epidermal growth factor receptors [7, 8], and human epidermal growth factor receptor-2 [9-13] are being developed for ovarian cancer therapy. Farletuzumab is a humanized IgG1 mAb that targets human folate receptor $\alpha$, which is highly expressed in most epithelial ovarian cancers [3]. The Phase 3 study of farletuzumab in combination with carboplatin and abraxane in ovarian cancer patients is currently recruiting participants.

CA125 is a repeating peptide epitope of the membrane-spanning mucin MUC16, which is highly expressed on the cell surface of ovarian cancer cells, mesotheliomas and other malignant tumors. In addition to malignant tissues, MUC16 is also expressed in normal epithelia of the endometrium, trachea, and cornea [14-16]. In normal tissues MUC16 is believed to contribute to fetal development, acts as a barrier to trophoblast adherence and bacterial infection and, through its interactions with cytoskeletal proteins, plays a role in the formation of microplicae in the corneal epithelium [15-19]. In ovarian tumors, MUC16 plays an important immunosuppressive role by blocking the cytotoxicity of natural killer (NK) cells and also by facilitating peritoneal metastasis of malignant ovarian cancer cells [20-24].

Mesothelin is a tumor differentiation antigen expressed at high levels in mesotheliomas, as well as ovarian, pancreatic and lung cancers [25-26]. Our recent studies have indicated that mesothelin may be a potential therapeutic target in primary liver cancer, particularly cholangiocarcinoma [27]. MUC16 binds to mesothelin and mediates attachment of ovarian cancer cells to the peritoneal walls [23, 28-30]. The MUC16-mesothelin interaction mediates cancer cell adhesion $[28,30]$, indicating that the interaction may possibly play an important role in the implantation and spread of tumors. Furthermore, we have recently identified that a functional domain in mesothelin from residues 296 - 359, named IAB, is required and sufficient to bind MUC16 [30]. Because MUC16 is poorly internalized, immunoconjugates or drug conjugates that act inside the cells may be less effective. Efforts have focused on making mouse mAbs to MUC16 that are currently being evaluated in clinical (mAb B43.13 or Oregovomab or OVARex ${ }^{\circledR}$, ViRexx Medical Corp., Edmonton, Canada) [1, 31-33] and preclinical studies (mAbs 11D10 and 3A5, Genentech, South San Francisco, CA) [34]. However, a human $\mathrm{mAb}$ against MUC16 has never been identified. Because of its lower immunogenicity in patients, a fully human protein is the most desirable format for clinical applications. We postulate that a more desirable $\mathrm{mAb}$ targeting MUC16-expressing tumors would be a human IgG that can functionally block the MUC16-mesothelin interaction, as well as elicit internalization-independent anti-tumor activity.

In recent years, human IgG Fc fusion proteins have emerged as a credible alternative to mAbs in drug development [3, 35]. Most successful therapeutic antibodies or Fc fusion proteins target receptor and ligand interactions. Etanercept (Enbrel) is the first successful example of using a soluble receptor (TNFRII)-Fc fusion protein as a therapeutic drug. The US Food and Drug Administration has approved four Fc fusion proteins: Etanercept (Enbrel, TNF $\alpha$ R), Alefacept (Amevive, LFA-3), Abatacept (Orencia, hCTLA-4) and Rilonacept (Binding domains of IL-1R and IL-1RacP). Many more Fc fusion proteins are currently being evaluated in clinical trials [3]. Aflibercept and AMG 386, two Fc fusion proteins designed to interfere with tumor angiogenesis, are currently being evaluated in Phase 3 clinical studies to treat ovarian cancer. Aflibercept contains the extracellular domains of VEGFR1 and VEGFR2. AMG 386 is a "peptibody" composed of a peptide that binds angiopoietin- 1 and -2 proteins.

Here, we report the generation of a novel human Fc fusion immunoadhesin (named HN125) that consists of IAB, the MUC16-binding domain of mesothelin, fused to the $\mathrm{N}$ terminus of the Fc portion of a human antibody IgG1. We show that HN125 has high and specific affinity for MUC16-expressing human ovarian cancer cells, has the ability to significantly inhibit the MUC16-mesothelin interaction on cancer 
cells, and elicits strong antibody-dependent cell mediated cytotoxicity against cancer cells. Our results indicate that HN125 has therapeutic potential as a novel agent for treating ovarian cancer and other MUC16-expressing malignant tumors. This is the first report of a human immunotherapeutic agent that binds MUC16-expressing cancer cells and kills them without the aid of radioisotopes or cytotoxic drugs.

\section{MATERIAL AND METHODS}

\section{Cell Culture}

A431/H9 is an A431 human epithelial carcinoma cell line that has been genetically engineered in our lab to over-express human mesothelin [36]. A431/H9 cells were grown in DMEM with $10 \%$ fetal bovine serum (FBS) (Hyclone, Thermo Scientific, Pittsburgh, PA), 1\% L-glutamine, 1\% penicillin, 1\% streptomycin (Invitrogen, Carlsbad, CA) and G418 $(700 \mu \mathrm{g} / \mathrm{mL})$ (Invitrogen). ECC-1 is an endometrial cancer cell line and OVCAR3 is an epithelial ovarian tumor cell line, and both were purchased from ATCC (Manasas, VA). OVCAR3 (ovarian) cells were grown in DMEM with $10 \%$ FBS, $1 \%$ L-glutamine, $1 \%$ penicillin, $1 \%$ streptomycin (Invitrogen) and with human insulin (10 $\mu \mathrm{g} / \mathrm{mL}$ ) (Eli Lilly, Indianapolis, IN). NCI-H226 and YOU (mesothelioma) cells were grown in RPMI 1640 (Invitrogen) supplemented with 10\% FBS (Hyclone), $1 \%$ penicillin/streptomycin, and $1 \%$ l-glutamine (Invitrogen). ECC-1 cells were cultured in RPMI 1640 media (Invitrogen) containing 10\% fetal calf serum. Cells were confirmed to be negative for mycoplasma.

\section{Construction of Expression Plasmid}

PCR amplification of IAB from pMH118 [30] was performed using primers IAB-F(EcoRI): 5'-GAATTCGATATCGGAAGTGGAGAAGACAGC CTGTCCT-3' and IAB-R(BglII): 5'-AGATCTGAGC TCATCCAGTTTATGCTTTAGG-3'. The PCR product was purified and digested with EcoRI and BglII (New England Biolabs, Ipswich, MA), then ligated with pFUSE-hIgG1-Fc2 (Invivogen, San Diego, CA) linearized with EcoRI and BglII. The resultant vector was reamplified for the IAB-hFc (Fc portion of a human antibody IgG1) part using primers IL2-F: 5'-TCGACACGTGTGATCAGATAGGGCCACCATG TACAGGATGCAACTCCTGTCT-3' and hFc-R: 5'TCTAGAGCGGCCGCGATACTCATTTACCCGGAG ACAGGGAGAG-3', The IAB-hFc PCR fragment was then digested with $P m \mathrm{I}$ and NotI, and inserted into the vector $p V R C 8400[37,38]$. The final plasmid is named pMH142 (Figure 1A).
A
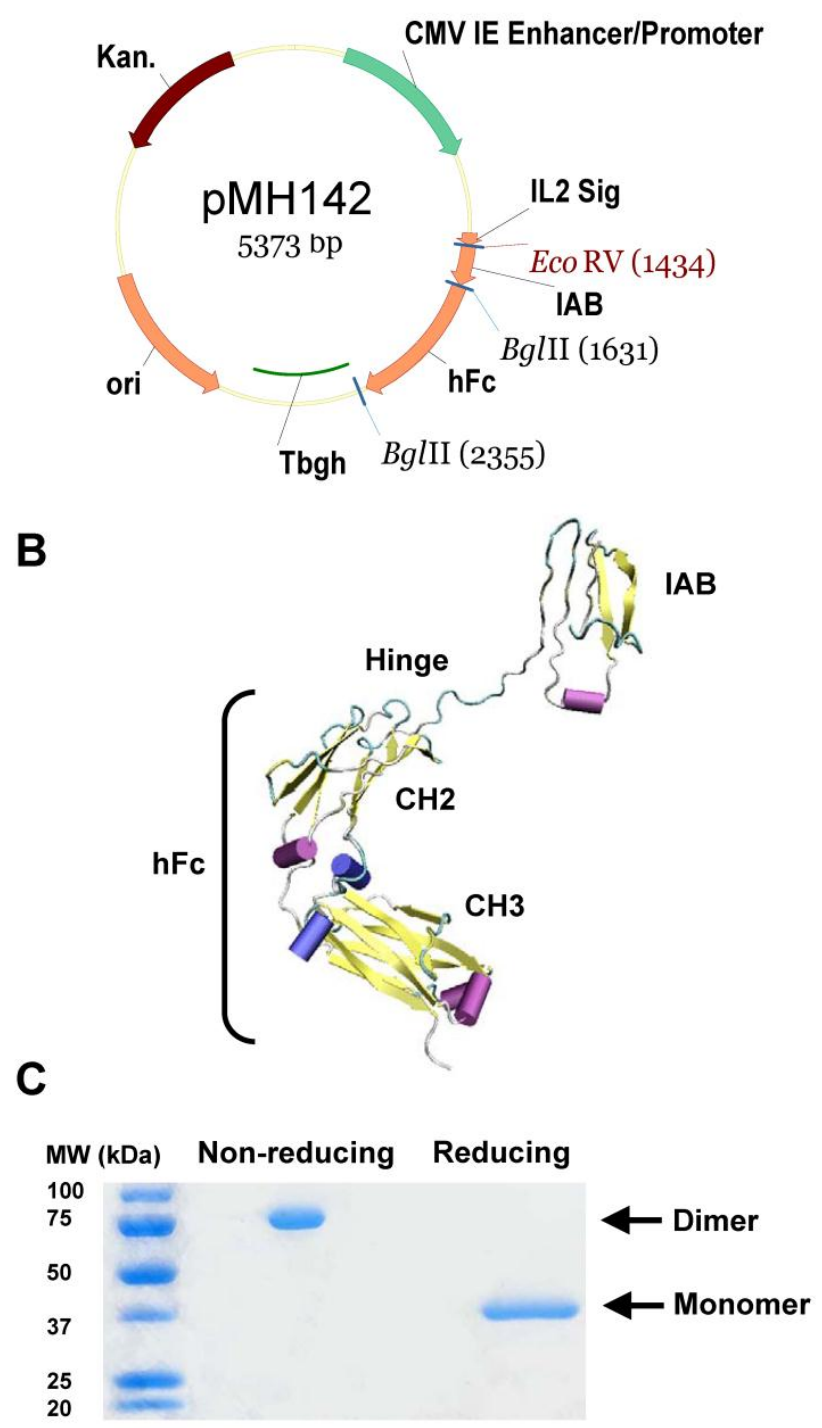

Figure I. Generation of immunoadhesin HNI25. A. Diagram of expression plasmid pMHI 42 for $\mathrm{HN} 125$ expression in human $\mathrm{HEK}-293 \mathrm{~F}$ cells as $\mathrm{NH}_{2}$-terminal fusions to human IgGI $\mathrm{Fc}(\mathrm{hFc})$ (Hinge-CH2-CH3). CMV IE Enhancer/Promoter: human cytomegalovirus immediate-early enhancer/promoter; IL2 Sig: IL2 signal peptide; Tbgh: poly A bovine growth hormone tail; ori: origin of replication; Kan.: kanamycin resistance. B. The structural model of HNI 25. $I A B$ is the functional binding domain in mesothelin for MUCI6. $\mathrm{CH} 2$ and $\mathrm{CH} 3$ : second and third constant domains of a human IgGI, respectively. C. SDS-PAGE analysis of HNI 25. Molecular weights: non-reduced dimers ( $\sim 75 \mathrm{kDa})$ and reduced monomers $(\sim 37 \mathrm{kDa})$. 


\section{Expression and Purification of HNI 25}

Using 293fectin, $30 \mu \mathrm{g}$ of $\mathrm{pMH142}$ plasmid was transiently transfected into $3 \times 10^{7}$ HEK-293F cells (Invitrogen) and kept in $30 \mathrm{~mL}$ of FreeStyle expression medium (Invitrogen) in a $125-\mathrm{mL}$ spinner flask on a stirring platform at $75 \mathrm{rpm}$ (CELLSPIN system; Integra, Chur, Switzerland) in a humidified atmosphere containing $8 \% \mathrm{CO}_{2}$ at $37^{\circ} \mathrm{C}$. After three days, the medium was collected after centrifugation, replaced for an additional 3-4 days, and collected again. Pooled supernatants were then processed and Fc fusion protein (HN125) was purified using a 1-mL recombinant Protein A Hi-Trap column (GE Healthcare, Piscataway, NJ) on an AKTA Explorer Chromatography system (GE Healthcare) as described [39]. The quality and quantity of HN125 were determined by SDS-PAGE and $\mathrm{A}_{280}$ absorbance on a Nanodrop (Thermo Scientific/Nanodrop, Wilmington, DE), respectively.

\section{Structural Modeling of the HN I 25 Immunoad- hesin}

The structural model of HN125 was generated by molecular modeling using I-TASSER [40] (http:/ / zhanglab.ccmb.med.umich.edu/I-TASSER).

Molecular models were viewed and analyzed using VMD (http://www.ks.uiuc.edu/Research/vmd/) as described [41].

\section{Flow Cytometry and Binding Affinity}

OVCAR3 cells were used as MUC16-positive cells. A431/H9 cells were used as negative controls. To detect binding between HN125 and target cells, flow cytometry was performed on a FACSCalibur (BD Biosciences, San Jose, CA). First, 0.5-1 x $10^{6}$ cells were incubated with $5 \mu \mathrm{g} / \mathrm{mL}$ of HN125 for 1 hour. The binding was then detected with a 1:200 goat anti-human IgG conjugated with Alexa488 (Sigma, St Louis, MO) and incubated for 30 minutes. The binding affinity was determined using Prism (version 5) for Windows (GraphPad Software, La Jolla, CA).

\section{Immunohistochemistry}

Formalin-fixed and paraffin-embedded tissue blocks from 15 patients with ovarian cancer were retrieved from the files of the Armed Forces Institute of Pathology. Consecutive sections at 5-7 $\mu \mathrm{m}$ thickness were prepared and placed on positively charged slides. Sections were deparaffinized with three-changes of xylene, and washed with descending concentrations of alcohol and water, and subjected to antigen retrieval following our previously published protocol [42]. Immunostaining was carried out as previously described [43] with HN125 and an isotype control human IgG (Southern Biotech, Birmingham, $\mathrm{AL}$ ). The secondary antibody conjugated with peroxidase, diaminobenzidine and 3-amino-9-ethylcarbazole chromogen kits were obtained from Vector Laboratories (Burlingame, CA). To assess the specificity of the immunostaining, different negative controls were used, including the substitution of the primary antibody with the same isotype or pre-immune serum of the antibody, and omission of the secondary antibody. In addition, the immunostaining procedure was repeated at least twice using the same protocol and under the same conditions. Immunostained sections were independently evaluated by two investigators. A given cell was considered immunoreactive if distinct immunoreactivity was consistently seen in its cytoplasm, membrane, or nucleus, while all negative controls lacked distinct immunostaining.

\section{Heterotypic Cell Adhesion Assay}

OVCAR3 cells $\left(1 \times 10^{5}\right)$ were seeded in triplicate in 96-well plates and incubated overnight at $37^{\circ} \mathrm{C}, 5 \%$ $\mathrm{CO}_{2}$. A431/H9 cells were trypsinized the next day and labeled with CellTracker Red CMTPX Dye (Invitrogen) at a concentration of $5 \mu \mathrm{M}$ for 15 minutes following manufacturer's instructions. The OVCAR3 monolayer cells were washed once with $200 \mu \mathrm{L}$ of $10 \%$ complete RPMI and incubated for 1 hour on ice with different concentrations of HN125 or CD30-hFc as a negative control. Labeled $\mathrm{H} 9$ cells $\left(2 \times 10^{5}\right)$ were then added to each well for 1 hour on ice. Wells were washed five times with $200 \mu \mathrm{L}$ PBS by gently inverting the plate on paper towels. Representative pictures of each well were taken using a Zeiss Axio Observer Z1 microscope (Carl Zeiss MicroImaging, Inc., Thornwood, NY), and images were collected with Axiovision software (Carl Zeiss MicroImaging, Inc.). The average intensity of fluorescence was measured using ImageJ software (National Institutes of Health, Bethesda, MD).

\section{Epitope Mapping}

To map the mesothelin binding domain on MUC16, two methods of flow cytometry were used. In the first method, FACS (FACS Calibur) was performed by first incubating OVCAR3 cells $\left(0.5-1 \times 10^{6}\right)$ with different concentrations of the mouse mAbs OC125 (Invitrogen) and M11-like (Invitrogen) for 30 minutes in 5\% FACS buffer before HN125 was added at $0.1 \mu \mathrm{g} / \mathrm{mL}$ for 1 hour. The binding was then detected with a 1:200 goat anti-mouse IgG conjugated with PE (Sigma) and incubated for 30 minutes. In the second method, FACS was performed by incubating OVCAR3 cells $\left(0.5-1 \times 10^{6}\right)$ with different concentra- 
tions of HN125 before adding $0.1 \mu \mathrm{g} / \mathrm{mL}$ of OC125 and M11-like antibodies. The binding was then detected with a 1:200 goat anti-human IgG conjugated with FITC (Sigma).

\section{Antibody-dependent Cell Cytotoxicity Assay}

The antibody-dependent cell cytotoxicity (ADCC) assay was performed on OVCAR3 cells using human peripheral blood mononuclear cells (PBMC). PBMC (Department of Transfusion Medicine, NIH Clinical Center, Bethesda, MD) were isolated by diluting $15 \mathrm{~mL}$ of human buffy coat with equal volumes of PBS with 2 mM EDTA and serum free DMEM up to a total of $40 \mathrm{~mL}$. Ten $\mathrm{mL}$ of the diluted buffy coat were then layered gently on $3 \mathrm{~mL}$ of Ficoll and centrifuged at $900 \times \mathrm{xg}$ for 30 minutes at room temperature. The layer of PBMC was removed and diluted again to $4 \mathrm{x}$ volume with serum free DMEM before it was centrifuged and washed twice more with DMEM-all at 400xg for 10 minutes at room temperature. PBMC were mixed with trypsinized OVCAR3 cells $\left(1 \times 10^{4}\right.$ per well) at a ratio of 100:1. The mixture was incubated for $\sim 20$ hours at standard cell culture conditions. A non-specific human IgG (Southern Biotech) was used as a negative control. The viability of OVCAR3 cells was measured using a lactose dehydrogenase (LDH) detection kit (Roche Applied Science, Branford, CT) per manufacturer's instructions.

\section{ADCC Using Purified NK Cells}

NK cells were isolated from the peripheral blood of healthy donors using the RosetteSep Kit (StemCell Technologies, Vancouver, Canada). For the cytotoxicity assays, suspensions of OVCAR3 or ECC- 1 cells were labeled with ${ }^{51} \mathrm{Cr}$. The target cells $\left(1 \times 10^{5}\right)$ were incubated with the NK cells at different effector:target concentrations in the presence or absence of human IgG or HN125. After incubation for 4 hours, the ${ }^{51} \mathrm{Cr}$ released in the media was measured and lysis percentage was measured as described previously [20, 44].

\section{Statistical Analysis}

Statistical analysis was performed with Prism (version 5) for Windows (GraphPad Software). Raw data were analyzed by "analysis of variance" with Dunnett's and Newman-Keuls multiple comparison post tests. $p$ values $<0.05$ were considered statistically significant.

\section{Results}

\section{Expression and Purification of HN I 25}

We have identified the IAB region, consisting of 64 amino acids (EVEKTACPSGKKAREIDESLIFYK
KWELEACVDAALLATQMDRVNAIPFTYEQLDVLK HKLDEL) at the N-terminal of cell surface mature mesothelin, as the minimum fragment required for complete binding activity to MUC16 [30]. In the present study, we constructed a $\mathrm{hFc}$ protein that joins $\mathrm{IAB}$, the functional binding domain for MUC16, with the human IgG1 Fc fragment, containing $\mathrm{CH} 2$ and $\mathrm{CH} 3$ domains, at the hinge region (plasmid pMH142, Figure 1A). The hinge may serve as a flexible spacer between IAB and Fc, allowing each part of the molecule to function independently. We used a signal peptide from interleukin-2 (IL2). The predicted structure of this hybrid protein (named HN125) is shown as a monomer in Figure 1B.

HEK-293F cells were transfected with pMH142. Transfectants were first analyzed for secreted protein in the supernatant by ELISA. Since HN125 contained the human IgG1 Fc fragment, the Fc fusion protein in the supernatant was successfully purified in one step by affinity chromatography using protein A Sepharose. The purified HN125 was then analyzed by SDS-PAGE. Because the Fc region of human IgG1 introduced into the $\mathrm{Fc}$ fusion protein contained a hinge region, HN125 is expected to form an internal S-S linked dimer. HN125 showed a band of dimer size $(\sim 75 \mathrm{kDa})$ under non-reducing conditions, indicating the dimeric properties of HN125 (Figure 1C). A single band of monomer size $(\sim 37 \mathrm{kDa})$ was found under reducing conditions. The purity was above $95 \%$. The yield was over $100 \mu \mathrm{g} / \mathrm{mL}$ of culture supernatant.

\section{High Affinity Binding of HNI 25 on Cancer Cells}

The binding of HN125 to membrane-bound MUC16 on cancer cells was examined by flow cytometry. All the cancer cell lines (A431/H9, OVACR3, NCI-H226 and YOU) express mesothelin on the cell surface, while only OVCAR3 and YOU cells express MUC16 [30, 36, 39]. As shown in Figure 2 (A-D), HN125 specifically bound to the MUC16-positive ovarian cancer cells (OVCAR3) and mesothelioma cells (YOU), but not to the MUC16-negative A431/H9 and NCI-H226 cancer cells, indicating excellent specificity of HN125 for cancer cell-associated MUC16 molecules. We have also tested the binding of HN125 on additional four MUC16-negative cancer cell lines, no signal was found in any of these lines (data not shown). OVCAR3 cells showed a 600-fold increase in MUC16 detection. YOU cells had around ten-fold less MUC16 expression on the cell surface. Interestingly, HN125 showed clear and strong staining on YOU cells, indicating high binding affinity of HN125 for MUC16-positive cancer cells including those with low MUC16 expression. To measure the binding affinity of 
HN125 on cancer cells, we made the binding saturation curve and Scatchard Plot (Figure 2E and 2F). The $K_{d}$ of HN125 on OVCAR3 cells was $13 \mathrm{nM}$.

A human micro-papillary ovarian tumor was immunostained with HN125 (Figure 3). In the presence of HN125, strong and uniform cytoplasmic immunoreactivities were observed in a vast majority of the tumor cells, while weak and diffuse staining was occasionally found only in some individual or clusters of cells with the isotype control. The expression and subcellular localization of the MUC16 epitope recognized by HN125 appeared to be correlated with tumor progression. Some cell clusters near the luminal face showed either no or nuclear immunostaining. Interestingly, nearly all tumor cells in the basal-lateral face showed strong immunoreactivities with HN125.
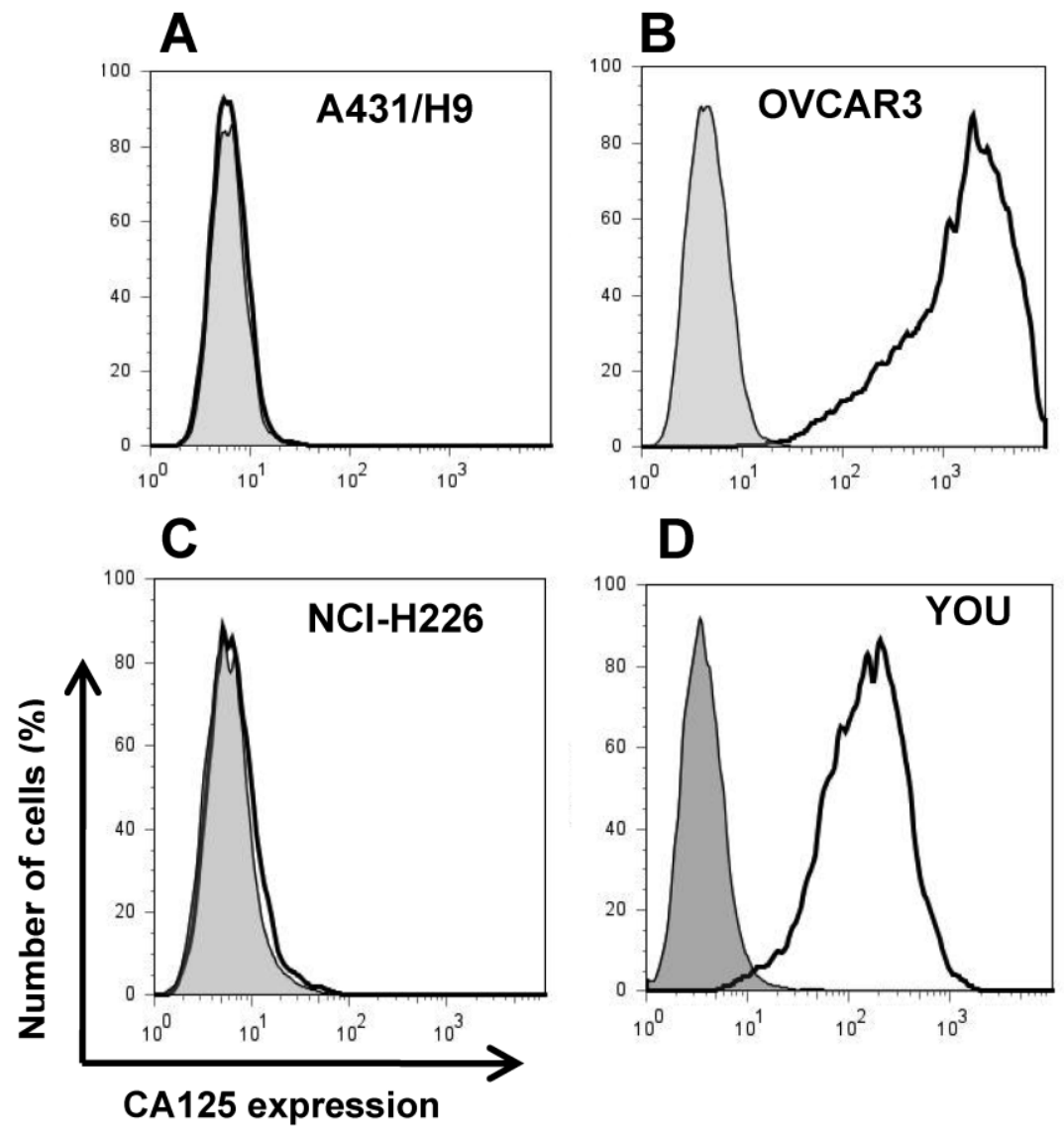

E

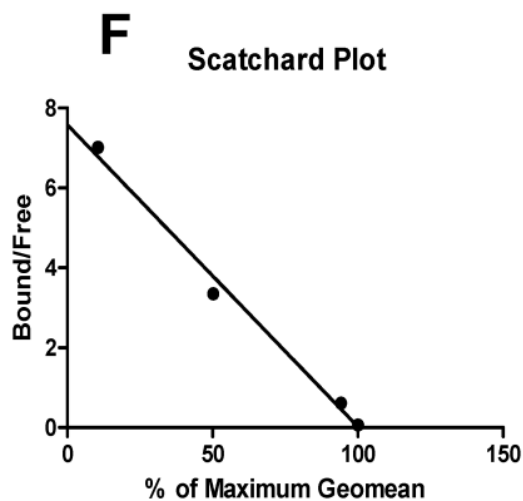

Figure 2. The binding of HNI 25 to MUCI6 on cancer cells. A43I/H9 (A), OVCAR3 (B), NCl-H226 (C) and YOU (D) cells were probed with HNI25 (solid dark line). Dark gray shading: secondary antibody control. Each cell line and its GeoMean value: A43 I/H9 (6), OVCAR3 (I229), NCl-H226 (6) and YOU (I37). GeoMean of the secondary antibody control was about 6. The binding saturation curve $(E)$ followed by Scatchard analysis $(F)$ showed that the $K_{D}$ of HNI 25 to OVCAR3 cells was $13 \mathrm{nM}$. 

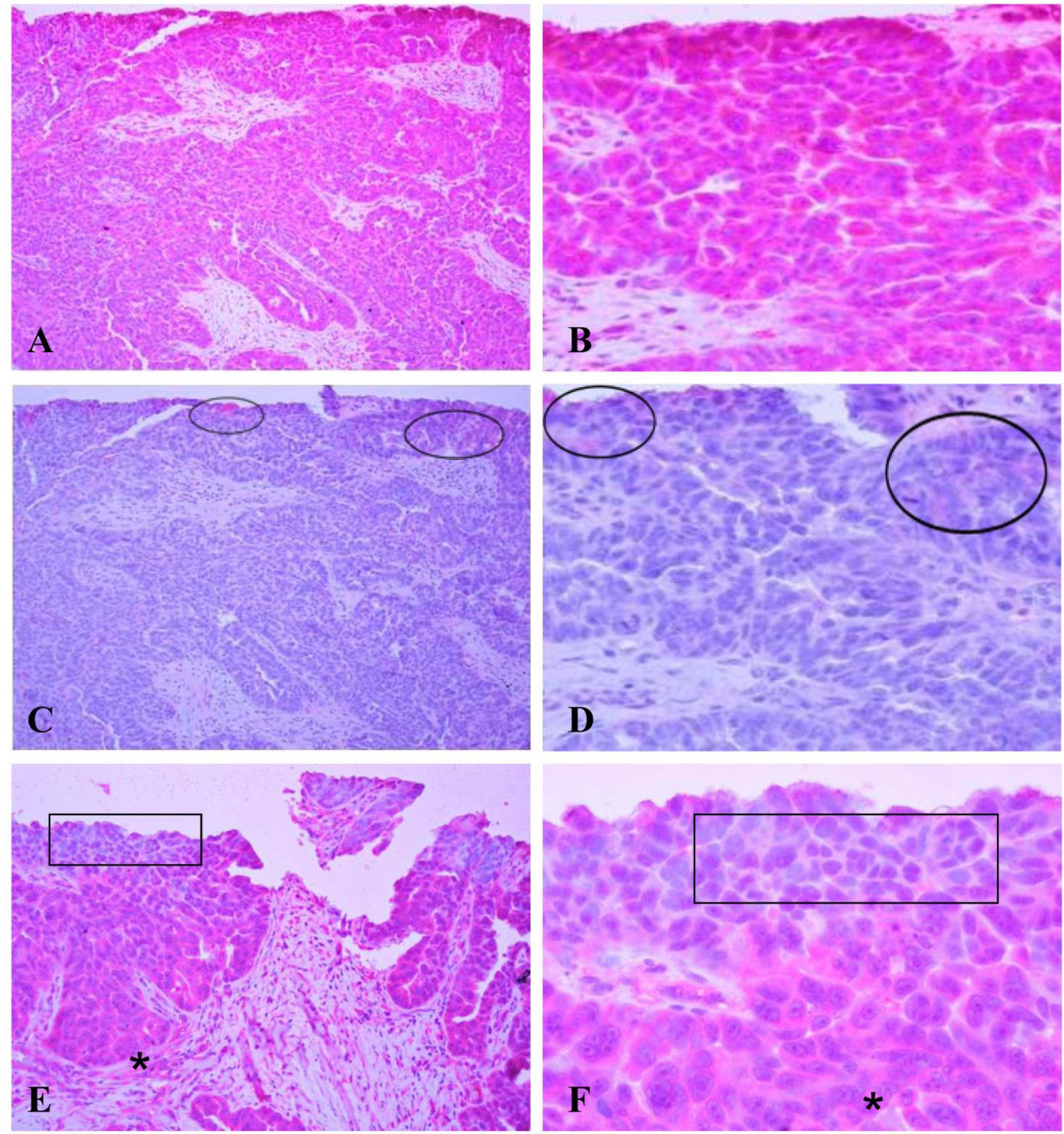

Figure 3. Immunostaining of $\mathrm{HNI} 25$ on human ovarian tumors. A human micro-papillary ovarian tumor was immunostained with HNI25 (A-B; E-F) and an isotype human lgG control (C-D). Circles identify weak and diffuse staining occasionally found in some individual or clusters of cells with the isotype control. Squares in $E$ and $F$ show cells of the superficial layers (luminal face) with no, weak, or nuclear expression of the protein. Asterisks show cells of the deeper layers (baselateral face) with more intense and cytoplasmic expression of the protein. A, C, and E: I00X. B, D, and F: a higher magnification (400X) of $\mathrm{A}, \mathrm{C}$, and $\mathrm{E}$, respectively.

\section{Mesothelin Binding Site on MUCI6}

The well-characterized OC125 mAb was used to investigate the mesothelin binding domain on MUC16 by flow cytometry. OC125 developed by Bast et al. reacts with CA125, an epitope of MUC16 present on the surface of most epithelial ovarian tumors [45]. Figure 4 shows that when HN125 or full-length mesothelin was incubated with OVCAR3 cells first, the interaction between OC125 and MUC16 was reduced. The difference was slightly larger using full-length 
mesothelin than using HN125. No difference was found using another anti-CA125 mouse mAb M11 (data not shown). The marginal inhibition observed with OC125 indicated that the HN125 binding epitope may be different than the OC125 binding epitope (CA125). Previous studies indicated that the mesothelin-binding site on CA125 is N-glycan dependent [23]. The precise binding site of mesothelin on MUC16 remains elusive.

\section{Heterotypic Cell Adhesion Assay}

The heterotypic cell adhesion assay was performed to determine if HN125 could inhibit the interaction between cancer cells expressing MUC16 and those expressing mesothelin. HN125 at $100 \mu \mathrm{g} / \mathrm{ml}$ was able to significantly reduce, by approximately $80 \%$, the adherence of mesothelin-positive A431/H9 cells onto the MUC16-positive OVCAR3 monolayer (Figure 5). At $10 \mu \mathrm{g} / \mathrm{mL}, \mathrm{HN} 125$ was able to block $\sim 50 \%$ of the heterotypic adhesion.
MSLN
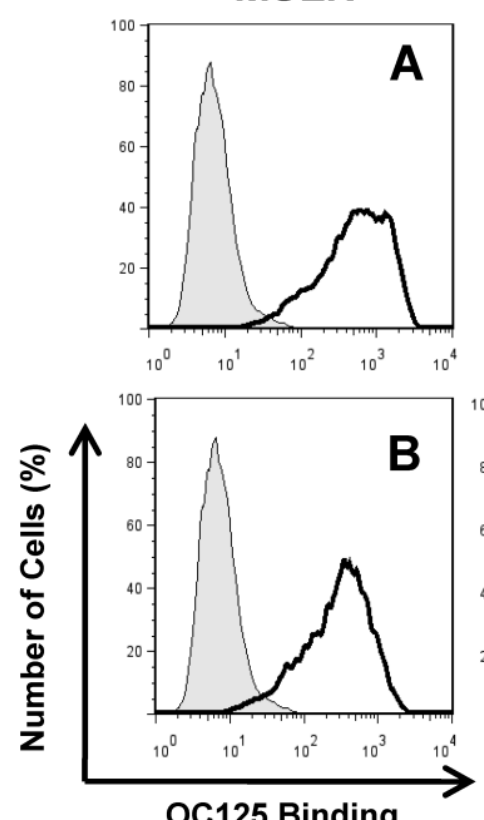

OC125 Binding
HN125
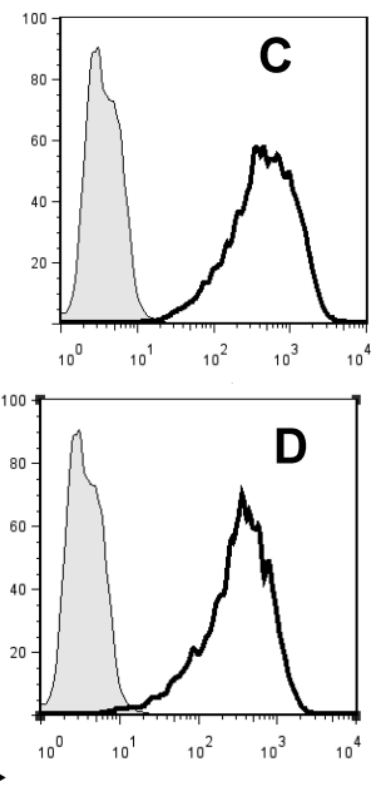

Figure 4. Inhibition of the HNI 25 binding to MUCI 6 by OCI25. OVCAR3 cells were probed with an anti-CAI25 $\mathrm{mAb} O \mathrm{OCI} 25$ (solid dark line) or an irrelevant isotype mAb control (light gray shading). Cells were incubated with 0 (A, C) or $10 \mu \mathrm{g} / \mathrm{mL}$ of full-length mesothelin (MSLN) (B) or HNI25 (D) before OCI25 was added. Their GeoMean values: A, 480; B, 262; C, 4I0; D 287. GeoMean of the isotype antibody control was about 5.0.

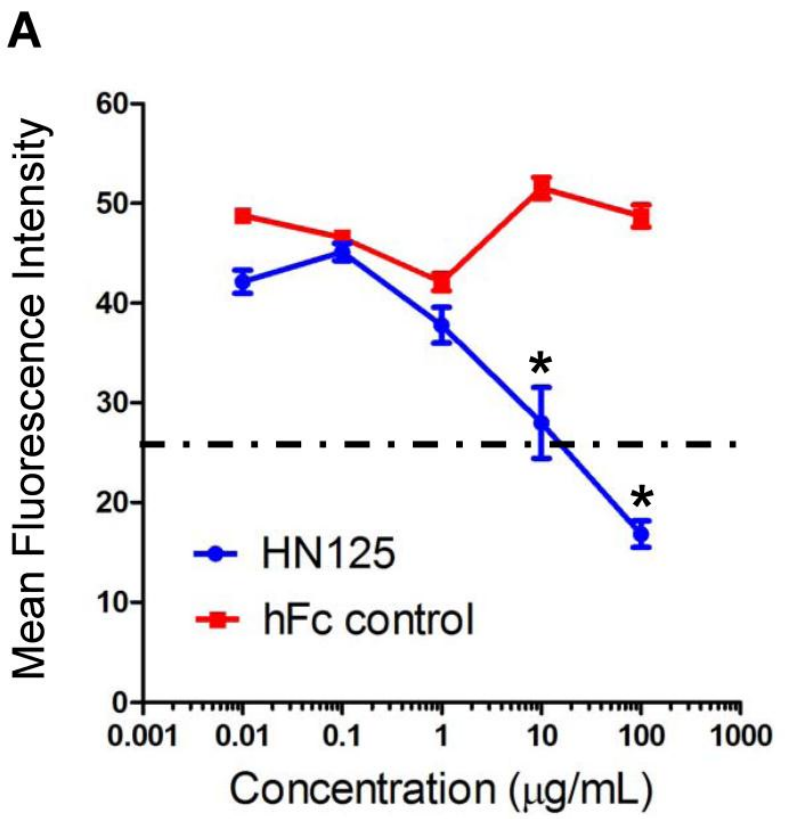

B HN125 hFc control
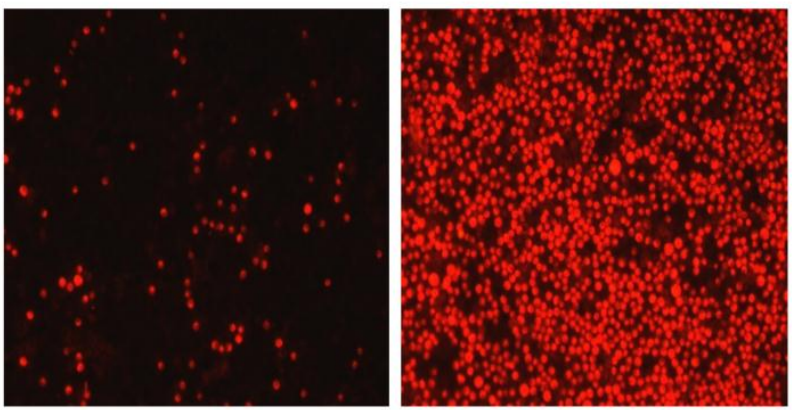

Figure 5. Inhibition of the MUCI6-mesothelin mediated heterotypic cancer cell adherence. HNI 25 was examined in a heterotypic cell adhesion assay using OVCAR3 and A43 I/H9 cell lines. A. The mean intensity of each image was then analyzed using ImageJ software. The heterotypic cell adhesion between cells expressing mesothelin and MUCI6 was inhibited in the presence of $10-100 \mu \mathrm{g} / \mathrm{mL}$ of HNI25 $(p<0.0 \mathrm{I})$. B. Microscopic images of cell adhesion assays in the presence of $100 \mu \mathrm{g} / \mathrm{mL}$ of $\mathrm{HNI} 25$ or hFc control. hFc control: CD30-Fc.

\section{Anti-tumor Activity}

To determine if the human IgG Fc domain of HN125 was functionally able to direct ADCC toward antigen expressing target cells, we tested HN125 on OVCAR3 cells. As shown in Figure 6A, using the PBMC from healthy donors, HN125 exerted significant ADCC activity by killing about 35\% OVCAR3 cells. No significant lysis of the targets was found 
when the assays were conducted with a human IgG control. When tested on MUC16-negative cells (A431/H9), no ADCC activity was found. We also tested HN125 on YOU cells and significant but moderate anti-tumor activity was found (data not shown).

NK cells are the major component of naïve PBMC that mediates target cell lysis via ADCC. Therefore, we tested the HN125-mediated lysis of MUC16-expressing tumor target OVCAR3 cells and ECC-1 cells by NK cells isolated from the peripheral blood of two healthy donors. As shown in Figure 6B and $6 \mathrm{C}$, using $\mathrm{NK}$ cells isolated from both donors, HN125 can elicit dose-dependent ADCC activity in both cell types, even OVCAR3 cells that are generally highly resistant to NK cell attack [23; Patankar et al. unpublished data]. Furthermore, the extent of lysis of OVCAR3 cells differs between NK cells isolated from different donors. Finally, we did not find significant complement-dependent cytotoxicity by HN125 (data not shown), suggesting that HN125 may primarily mediate tumor target killing via ADCC.

Figure 6. HNI25-mediated killing of CAI25-expressing cancer cells via ADCC. A. OVCAR3 cells were incubated with PBMC and either $10 \mu \mathrm{g} / \mathrm{mL}$ of $\mathrm{HNI} 25$ or a control human IgG. Percent of cell death was measured using a LDH kit. About $35 \%$ of target cells were killed when treated with HNI 25 ( $p$ values $<0.05$ ). B and C. NK cells isolated from peripheral blood of 2 healthy donors. OVCAR3 (B) and ECC-I (C) cells used as targets were loaded with ${ }^{51} \mathrm{Cr}$. Cell lysis was determined by the release of ${ }^{51} \mathrm{Cr}$. Lysis assays were conducted in the presence or absence of HNI25 as designated. Representative data shown is for effector:target ratio of 12:I. Each E:T was tested in triplicate in three separate experiments.

\section{DISCUSSION}

Recent studies have shown that MUC16 binds mesothelin [28]. The MUC16-mesothelin interaction mediates cancer cell adhesion, indicating that MUC16 and mesothelin may possibly play an important role in the implantation and metastatic spread of tumors. Therefore, there is an emerging interest to develop a drug to disrupt the interaction of MUC16 and mesothelin. In the present study, we report the development of immunoadhesin HN125, a functional domain-Fc fusion protein, with great potential as a novel cancer therapeutic by disrupting the important biological interactions involving cancer pathogenesis or metastasis, and delivering effector cells specifically to the tumor cells that over-express MUC16 at the cell
A

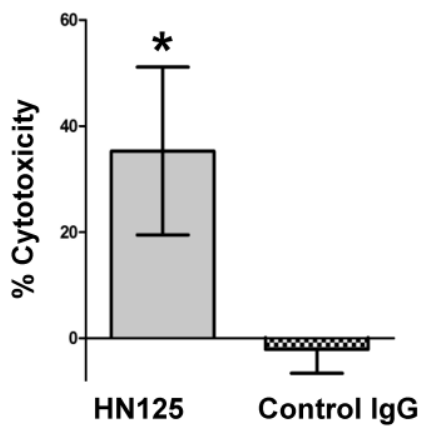

B

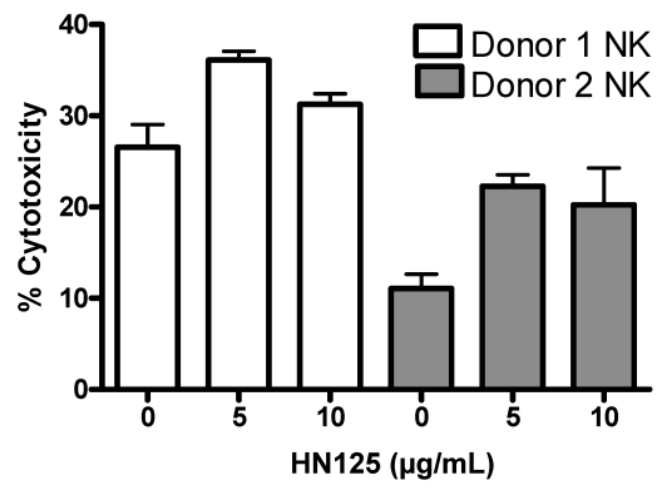

C

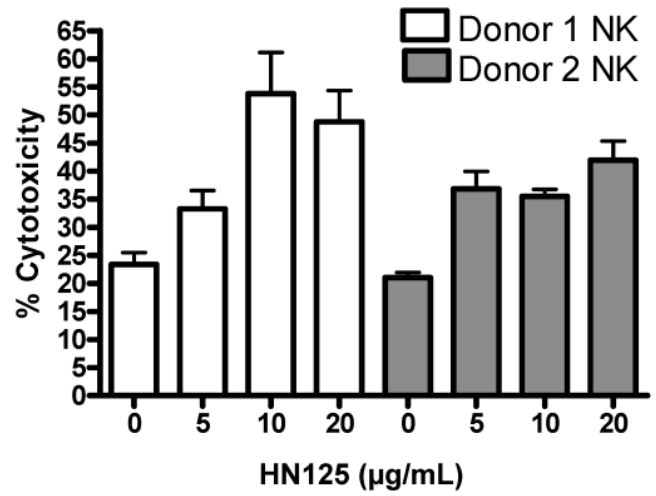

surface. HN125 is the first reported fully human immunotherapeutic agent specific for MUC16, which has the potential for cancer treatment and diagnosis.

In principle, the efficacy of anti-cancer antibody therapy may be mainly determined by the number of antigens on the cell surface and the rate of antigen internalization. A recent study evaluated mAb 11D10 for a non-repeating region of MUC16, and the antibody exhibited only partial activity against OVCAR3 cells in vitro and in OVCAR3 xenograft models [34]. Interestingly, the same research group found $\mathrm{mAb}$ $3 \mathrm{~A} 5$ against the mucin repeats of CA125, and that allowing multiple antibody bindings per CA125 molecule was more effective by increased binding of $3 \mathrm{~A} 5$ 
to cancer cells. Immunoconjugates or drug conjuates may enhance drug efficacy, however, due to its slow internalization, MUC16 is a poor target for the immunoconjugates or drug conjugates that act inside cancer cells.

Several mouse mAbs have been developed and have been evaluated in clinical and preclinical studies [1]. In a randomized, double-blind, placebo-controlled trial of stage III/IV ovarian cancer patients with a complete clinical response, Berek et al. treated 145 patients with oregovomab or a placebo [33]. The primary endpoint of the study was time to relapse (TTR), and median TTR was 24 months for oregovomab compared to 11 months for the placebo. In a follow-up study 5 years after randomization, they also reported on long-term outcomes for this patient population [46]. Median survival was 58 months for patients given oregovomab compared to 49 months for the placebo. In new preclinical studies, a drug conjugated mouse mAb 3A50 that targets CA125 repeats showed high efficacy in mouse models [34]. Due to low immunogenicity, fully human proteins are highly desired for cancer therapy. No human $\mathrm{mAb}$ has been identified to date. HN125 is the first human antibody-like immunoadhesin molecule targeting MUC16 with therapeutic potential for treating ovarian cancer, mesothelioma and other MUC16-expressing tumors. It should be interesting to directly compare HN125 with other anti-MUC16 antibodies and evaluate their anti-tumor activities in the future.

One concern about MUC16 as a therapeutic target is that soluble MUC16 in serum or in the peritoneal fluid of patients may inhibit the binding of HN125. Previous studies analyzed more than 30 samples of ascites derived from individual epithelial ovarian cancer patients and found the concentration of MUC16 in this fluid ranges from 1 to $10 \mathrm{nM}$ [23]. Since even $100 \mathrm{nM}$ of soluble MUC16 did not inhibit the mesothelin-MUC16 interaction on cells, it is reasonable to presume that soluble MUC16 in serum or peritoneal fluid would not neutralize HN125.

The mesothelin gene encodes a 71-kDa precursor protein that is processed to a $40-\mathrm{kDa}$ membrane-bound protein termed mesothelin and a 31-kDa shed fragment called megakaryocyte-potentiating factor (MPF) that is released from the cell [25]. We previously generated several forms of recombinant mesothelin proteins. We constructed the maltose-binding protein (MBP)-mesothelin fusion expression plasmid (pMH103) for the human mesothelin expression in E. coli [36]. We also made the full-length mesothelin expression plasmid (pMH107) and generated the A431/H9 cell line stably expressing recombinant human mesothelin and MPF proteins [36]. In a recent study, we constructed the rabbit IgG Fc-mesothelin fusion expression plasmids for human mesothelin (pMH113 or pMH113b) and mouse mesothelin (pMH117) expression in human HEK-293 cells [30]. In all of these constructs, mesothelin was fused to the $\mathrm{C}$ terminus of a native (MPF) or recombinant (MBP and Fc) partner protein. To generate an effective immunoadhesin, it is essential to fuse the functional domain (IAB) of mesothelin to the $\mathrm{N}$ terminus of the hinge region followed by the human $\operatorname{IgG} \mathrm{Fc}_{\mathrm{C}}(\mathrm{CH} 2$ and $\mathrm{CH} 3$ domains) which may mediate effector functions such as ADCC and CDC. The present study for the first time built a recombinant mesothelin protein at the $\mathrm{N}$ terminus of a fusion protein and the mesothelin retained its biological functions such as the MUC16 binding. Furthermore, the protein production yield was high (ranging from 100 to $500 \mu \mathrm{g} / \mathrm{mL}$ of HEK293 culture supernatant), indicating our HEK-293 expression method is feasible for large-scale protein production of HN125 for preclinical and clinical studies.

The present study used the cancer cells grown at the monolayer level for in vitro anti-tumor activity assays such as cell adherence inhibition and ADCC. We and others have shown that cancer cells cultured as monolayers exhibit less resistance to therapy than those grown in vivo and may be explained by "multicellular resistance", a mechanism for drug resistance attributed to cell-cell contacts, cell-matrix contacts, and the three-dimensional (3D) shape found in tumor tissue [47]. Future studies utilizing in vitro tumor spheroid models [47] and in vivo mouse models [48] are necessary to validate HN125 as a potential therapeutic agent for cancer therapy.

Our study reports HN125 as a novel immunoadhesin against tumor-associated MUC16. This approach is novel because it does not depend on generations of human mAbs, but rather it uses a functional domain involved in cancer cell adhesion. Because HN125 is entirely of human origin and has high affinity for its target, it is expected to be less immunogenic than murine $\mathrm{mAb}$ and to be more efficient in targeting MUC16-expressing tumors. Consequently, it should be further evaluated as a potential therapeutic reagent for the treatment of ovarian cancer and other MUC16-expressing cancers.

\section{ACKNOWLEDGMENTS}

We thank Dr. Gary J. Nabel (Vaccine Research Center, NIAID) for providing the pVRC8400 plasmid, NCI Flow Cytometry Core for assistance with flow cytometry, and the NIH Fellows Editorial Board for editorial assistance.

This work was supported in part by the Intramural Research Program of the NIH, National Cancer 
Institute, Center for Cancer Research, in part by an Ovarian Cancer Research Fund Individual Investigator Award to $\mathrm{MH}$, and in part by a Mesothelioma Applied Research Foundation Grant in Honor of Craig Kozicki to MH. This work was also possible because of the grants from the National Institutes of Health (1R41CA132520-01A2 and 1R21CA143616-01) to JPC and MSP. Dr. Mitchell Ho is a co-inventor on patents assigned to the United States of America, as represented by the Department of Health and Human Services, for the investigational products. The funders had no role in study design, data collection and analysis, decision to publish, or preparation of the manuscript. The content of this publication does not necessarily reflect the views or policies of the Department of Health and Human Services, nor does mention of trade names, commercial products, or organizations imply endorsement by the U.S. Government.

\section{Conflict of Interest}

There is no conflict of interest and the financial disclosure has been described in the Acknowledgements.

\section{References}

1. Frederick PJ, Straughn JMJr, Alvarez RD, Buchsbaum DJ. Preclinical studies and clinical utilization of monoclonal antibodies in epithelial ovarian cancer. Gynecol Oncol 2009;113: 384-390.

2. Jemal A, Siegel R, Xu J, Ward E. Cancer statistics, 2010. CA Cancer J Clin 2010;60: 277-300.

3. Reichert JM. Antibody-based therapeutics to watch in 2011. MAbs 2011;3: 76-99.

4. Monk BJ, Choi DC, Pugmire G, Burger RA. Activity of bevacizumab (rhuMAB VEGF) in advanced refractory epithelial ovarian cancer. Gynecol Oncol 2005;96: 902-905.

5. Monk BJ, Han E, Josephs-Cowan CA, Pugmire G, Burger RA. Salvage bevacizumab (rhuMAB VEGF)-based therapy after multiple prior cytotoxic regimens in advanced refractory epithelial ovarian cancer. Gynecol Oncol 2006;102: 140-144.

6. Burger RA, Sill MW, Monk BJ, Greer BE, Sorosky JI. Phase II trial of bevacizumab in persistent or recurrent epithelial ovarian cancer or primary peritoneal cancer: a Gynecologic Oncology Group Study. J Clin Oncol 2007;25: 5165-5171.

7. Berchuck GC, Rodriguez A, Kamel RK, Dodge JT, Soper DL, et al. Epidermal growth factor receptor expression in normal ovarian epithelium and ovarian cancer. I. Correlation of receptor expression with prognostic factors in patients with ovarian cancer. Am J Obstet Gynecol 1991;164: 669-674.

8. Scambia G, Benedetti Panici P, Battaglia F, Ferrandina G, et al. Significance of epidermal growth factor receptor in advanced ovarian cancer. J Clin Oncol 1992;10: 529-535.

9. Felip E, Del Campo JM, Rubio D, Vidal MT, Colomer R, et al. Overexpression of c-erbB-2 in epithelial ovarian cancer. Prognostic value and relationship with response to chemotherapy. Cancer 1995;75: 2147-2152.

10. Meden H, Marx D, Roegglen T, Schauer A, Kuhn W. Overexpression of the oncogene c-erbB-2 (HER2/neu) and response to chemotherapy in patients with ovarian cancer. Int J Gynecol Pathol 1998;17: 61-65.
11. Aigner A, Hsieh SS, Malerczyk C, Czubayko F. Reversal of HER-2 over-expression renders human ovarian cancer cells highly resistant to taxol. Toxicology 2000;144: 221-228.

12. Høgdall EV, Christensen L, Kjaer SK, Blaakaer J, Bock JE, et al. Distribution of HER-2 overexpression in ovarian carcinoma tissue and its prognostic value in patients with ovarian carcinoma: from the Danish MALOVA Ovarian Cancer Study. Cancer 2003;98: 66-73.

13. Camilleri-Broët S, Hardy-Bessard AC, Le Tourneau A, Paraiso $\mathrm{D}$, Levrel $\mathrm{O}$, et al. HER-2 overexpression is an independent marker of poor prognosis of advanced primary ovarian carcinoma: a multicenter study of the GINECO group. Ann Oncol 2004;15: 104-112.

14. Argüeso P, Spurr-Michaud S, Russo CL, Tisdale A, Gipson IK. MUC16 mucin is expressed by the human ocular surface epithelia and carries the H185 carbohydrate epitope. Invest Ophthalmol Vis Sci 2003;44: 2487-2495.

15. Blalock TD, Spurr-Michaud SJ, Tisdale AS, Heimer SR, Gilmore MS, et al. Functions of MUC16 in corneal epithelial cells. Invest Ophthalmol Vis Sci 2007;48: 4509-4518.

16. Davies JR, Kirkham S, Svitacheva N, Thornton DJ, Carlstedt I. MUC16 is produced in tracheal surface epithelium and submucosal glands and is present in secretions from normal human airway and cultured bronchial epithelial cells. Int J Biochem Cell Biol 2007;39: 1943-1954.

17. Gipson IK. Distribution of mucins at the ocular surface. Exp Eye Res 2004;78: 379-388.

18. Gipson IK, Blalock T, Tisdale A, Spurr-Michaud S, Allcorn S, et al. MUC16 is lost from the uterodome (pinopode) surface of the receptive human endometrium: in vitro evidence that MUC16 is a barrier to trophoblast adherence. Biol Reprod 2008;78: 134-142.

19. Govindarajan B, Gipson IK. Membrane-tethered mucins have multiple functions on the ocular surface. Exp Eye Res 2010;90: 655-663.

20. Patankar MS, Yu J, Morrison JC, Belisle JA, Lattanzio FA, et al. Potent suppression of natural killer cell response mediated by the ovarian tumor marker CA125. Gynecol Oncol 2005;99: 704-713.

21. Belisle JA, Gubbels JA, Raphael CA, Migneault M, Rancourt C, et al. Peritoneal natural killer cells from epithelial ovarian cancer patients show an altered phenotype and bind to the tumour marker MUC16 (CA125). Immunology 2007;122: 418-429.

22. Belisle JA, Horibata S, Gubbels JA, Petrie S, Kapur A, et al. Identification of Siglec-9 as the receptor for MUC16 on human NK cells, B cells, and monocytes. Mol Cancer 2010;9: 118.

23. Gubbels JA, Belisle J, Onda M, Rancourt C, Migneault M, et al. Mesothelin-MUC16 binding is a high affinity, N-glycan dependent interaction that facilitates peritoneal metastasis of ovarian tumors. Mol Cancer 2006;5: 50.

24. Gubbels JA, Claussen N, Kapur AK, Connor JP, Patankar MS. The detection, treatment, and biology of epithelial ovarian cancer. J Ovarian Res 2010;3: 8.

25. Hassan R, Ho M. Mesothelin targeted cancer immunotherapy. Eur J Cancer 2008;44: 46-53.

26. Ho M, Bera TK, Willingham MC, Onda M, Hassan R, et al. Mesothelin expression in human lung cancer. Clin Cancer Res 2007;13: 1571-1575

27. Yu L, Feng M, Kim H, Phung Y, Kleiner DE, et al. Mesothelin as a potential therapeutic target in human cholangiocarcinoma. J Cancer 2010;1: 141-149.

28. Rump A, Morikawa Y, Tanaka M, Minami S, Umesaki N, et al. Binding of ovarian cancer antigen CA125/MUC16 to mesothelin mediates cell adhesion. J Biol Chem 2004;279: 9190-9198.

29. Scholler N, Garvik B, Hayden-Ledbetter M, Kline T, Urban N. Development of a CA125-mesothelin cell adhesion assay as a 
screening tool for biologics discovery. Cancer Lett 2007;247: 130-136.

30. Kaneko O, Gong L, Zhang J, Hansen JK, Hassan R, et al. A binding domain on mesothelin for CA125/MUC16. J Biol Chem 2009;284: 3739-49.

31. Mobus VJ, Baum RP, Bolle M, Kreienberg R, Noujaim AA, et al. Immune responses to murine monoclonal antibody-B43.13 correlate with prolonged survival of women with recurrent ovarian cancer. Am J Obstet Gynecol 2003;189: 28-36.

32. Gordon AN, Schultes BC, Gallion H, Edwards R, Whiteside TL, et al. CA125- and tumor-specific T-cell responses correlate with prolonged survival in oregovomab-treated recurrent ovarian cancer patients. Gynecol Oncol 2004;94: 340-351.

33. Berek JS, Taylor PT, Gordon A, Cunningham MJ, Finkler N, et al. Randomized, placebo-controlled study of oregovomab for consolidation of clinical remission in patients with advanced ovarian cancer. J Clin Oncol 2004;22: 3507-3516.

34. Chen Y, Clark S, Wong T, Chen Y, Chen Y, et al. Armed antibodies targeting the mucin repeats of the ovarian cancer antigen, MUC16, are highly efficacious in animal tumor models. Cancer Res 2007;67: 4924-4232.

35. Huang C. Receptor-Fc fusion therapeutics, traps, and MIMETIBODY technology. Curr Opin Biotechnol 2009;20: 692-699.

36. Ho M, Hassan R, Zhang J, Wang QC, Onda M, et al. Humoral immune response to mesothelin in mesothelioma and ovarian cancer patients. Clin Cancer Res 2005;11: 3814-3820.

37. Barouch DH, Yang ZY, Kong WP, Korioth-Schmitz B, Sumida $\mathrm{SM}$, et al. A human T-cell leukemia virus type 1 regulatory element enhances the immunogenicity of human immunodeficiency virus type 1 DNA vaccines in mice and nonhuman primates. J Virol 2005;79: 8828-8834.

38. Ofek G, McKee K, Yang Y, Yang ZY, Skinner J, et al. Relationship between antibody 2F5 neutralization of HIV-1 and hydrophobicity of its heavy chain third complementarity-determining region. J Virol 2010;84: 2955-2962.

39. Ho M, Feng M, Fisher RJ, Rader C, Pastan I. A novel high-affinity human monoclonal antibody to mesothelin. Int J Cancer 2011;128:2020-2030.

40. Roy A, Kucukural A, Zhang Y. I-TASSER: a unified platform for automated protein structure and function prediction. Nat Protoc 2010;5: 725-738.

41. Ho M, Segre M. Inhibition of cocaine binding to the human dopamine transporter by a single chain anti-idiotypic antibody: its cloning, expression, and functional properties. Biochim Biophys Acta 2003;1638: 257-266.

42. Man YG, Tavassoli FA. A simple epitope retrieval method without the use of microwave oven or enzyme digestion. Appl Immunohistochem 1996;4: 139-141.

43. Hsiao YH, Siddiqui S, Man YG. Dual use of a single Wilms' tumor 1 immunohistochemistry in evaluation of ovarian tumors: a preliminary study of 20 cases. J Cancer 2010;1: 93-97.

44. Coligan JE, Kruisbeek AM, Marguiles DH, Shevach EM, Strober WE. Measurement of cytotoxic activity of NK/LAK cells. Curr Protoc Immunol 1996;3: Unit7-18.

45. Bast RCJr, Feeney M, Lazarus H, Nadler LM, Colvin RB, et al. Reactivity of a monoclonal antibody with human ovarian carcinoma. J Clin Invest 1981;68: 1331-1337.

46. Berek JS, Taylor PT, Nicodemus CF. CA125 velocity at relapse is a highly significant predictor of survival post relapse: results of a 5-year follow-up survey to a randomized placebo-controlled study of maintenance oregovomab immunotherapy in advanced ovarian cancer. J Immunother 2008;31: 207-214.

47. Xiang X, Phung Y, Feng M, Nagashima K, Zhang J, Broaddus VC, Hassan R, Fitzgerald D, Ho M. The development and characterization of a human mesothelioma in vitro 3D model to investigate immunotoxin therapy. PLoS One. 2011 Jan 31;6(1):e14640.

48. Feng M, Zhang J, Anver M, Hassan R, Ho M. In vivo imaging of human malignant mesothelioma grown orthotopically in the peritoneal cavity of nude mice. J Cancer. 2011 Mar 1;2:123-31. 